\title{
Ewing sarcoma of the testis: A case report and review of the literature
}

\author{
Javier Peinado-Serrano ${ }^{1,2}$ David Chinchón-Espino ${ }^{3}$ Michele Biscuola $^{3}$ and Maria José Ortiz-Gordillo ${ }^{1 *}$ \\ ${ }^{1}$ Department of Radiation Oncology, University Hospital Virgen del Rocio, Spain \\ ${ }^{2}$ Unit of Molecular Biology of Cancer, Biomedicine Institute of Seville (IBIS), Spain \\ ${ }^{3}$ Department of Pathology, University Hospital Virgen del Rocio, Spain
}

\begin{abstract}
Solid tumours in the inguinal scrotal area (inguinal, testicular and para-testicular) are infrequent in the clinic. After its observation, depending on factors such as age, time of evolution, metastatic disease and blood markers at diagnosis, clinicians oriented to one origin or other. However, this approach may lead to a wrong diagnosis and treatment approach.

In this publication, we report an extremely infrequent case of a Testicular Ewing Sarcoma in a 31-year-old patient. We include diagnostic and therapeutic approach, specially focused on the radiation oncologist point of view and also review the previous data reported in the literature.
\end{abstract}

\section{Background}

Testicular cancer represents $1 \%$ of male neoplasms and $5 \%$ of urological tumours, with 3-10 new cases occurring per 100,000 males/ per year in Western society [1].

According to the World Health Organisation classification, testicular tumours are classified in three main groups attending to histology and its origin: Germ cell tumours, sex cord/gonadal stromal tumours and Miscellaneous non-specific stromal tumour [2]. Ewing Sarcoma /PNET (primitive neuroectodermal tumour) of the testis could be included in any group, depending on the presence of other tumoral component associated.

From a biological point of view, Ewing Sarcoma family tumours (ESFT) are tumours of neural crest derivation that differentiate along a neuroendocrine lineage and are described as "small round cell tumours." All ESFT tumours are characterized by a balanced chromosomal translocation between the EWS gene (22q12) and genes which are considered as members of the E26 transformation-specific (ETS) family of transcription factors (FLI1 (11q24) and ERG (21q22)) [3,4].

Extraskeletal ewing sarcoma (EES), as our case, are commonly treated with surgery, chemotherapy and radiation, according to international treatment protocols [5]. In this sense, surgical resection with proper oncological margins is one of the mainstay of tumor control. According to recent publications (reporting data analysis from SEER and/or single/ multi institutional databases), the prognosis of EES compared to skeletal ones is equivalent [7], although some publications showed controversial results [6].

\section{Case report}

At December 2014, a 31-year-old man presented at our hospital (Urology department) with two-month history of a palpable mass located at the left testis with a progressive enlargement. At the time of presentation, vital signs were normal and there were no other symptoms or signs such as weight loss, fever or evidence of other nodule or masses in different anatomic places. The patient only complained of local slight discomfort.

Family history of a second cousin with testicular cancer (unknown histology)

There was no remarkable personal history of medical illness except for irritable bowel syndrome. Former smoker of 20 cigarettes a day for ten years till diagnosis. No alcohol consumption. On a physical examination, a soft, tender mass that measured approximately $4 \mathrm{~cm}$ in diameter was palpated in the left scrotal sac. Testicular ultrasonography (Figure 1) demonstrated the presence of a $4 \mathrm{~cm}$ round mass with significant increased blood flow. With the suspect of germ line tumor, the patient is subjected to an orchiectomy via a left inguinal canal approach on January 2015, with a complete removal of the testis, apparently without breakage or fragmentation of the mass, but without the guarantees of strict oncological surgery due to the first suspected diagnosis.

\section{Pathological diagnosis}

- Macroscopic description of orchiectomy: Mass of 3,6 x 2,7 cm which is $0.1 \mathrm{~cm}$ far from the tunica albuginea.

- Microscopic description (first diagnosis): informed as Spermatocytic Seminoma. TNM classification: pT1cN0cM0.

- Second review. Histological diagnosis based on morphological and immunostaining characteristics (Figure 2).

Correspondence to: Maria Jose Ortiz Gordillo, $\mathrm{PhD}$, Department of Radiation Oncology, University Hospital Virgen del Rocio, Manuel Siurot s/n. PC 41013, Seville, Spain. Tel: + 349550121 05, E- mail: mariaj.ortiz.sspa@juntadeandalucia.es key words: ewing sarcoma, primitive neuroectodermal tumor, testicular neoplasm, radiotherapy

Received: February 05, 2017; Accepted: February 25, 2017; Published: February 27,2017 


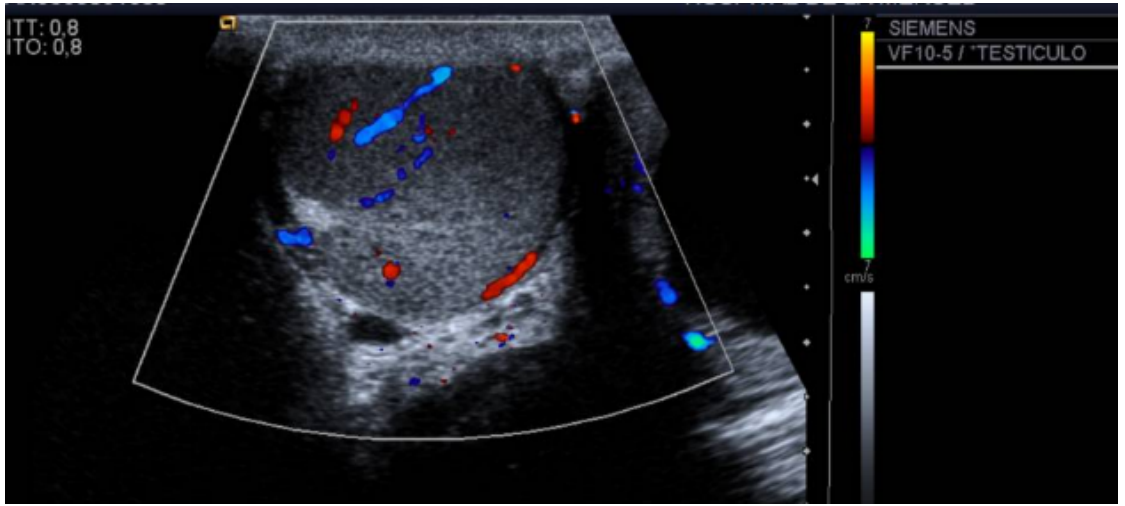

Figure 1. Diagnostic testicular ultrasound showing hyper vascularized testicular mass. Echo-Doppler technique is used.

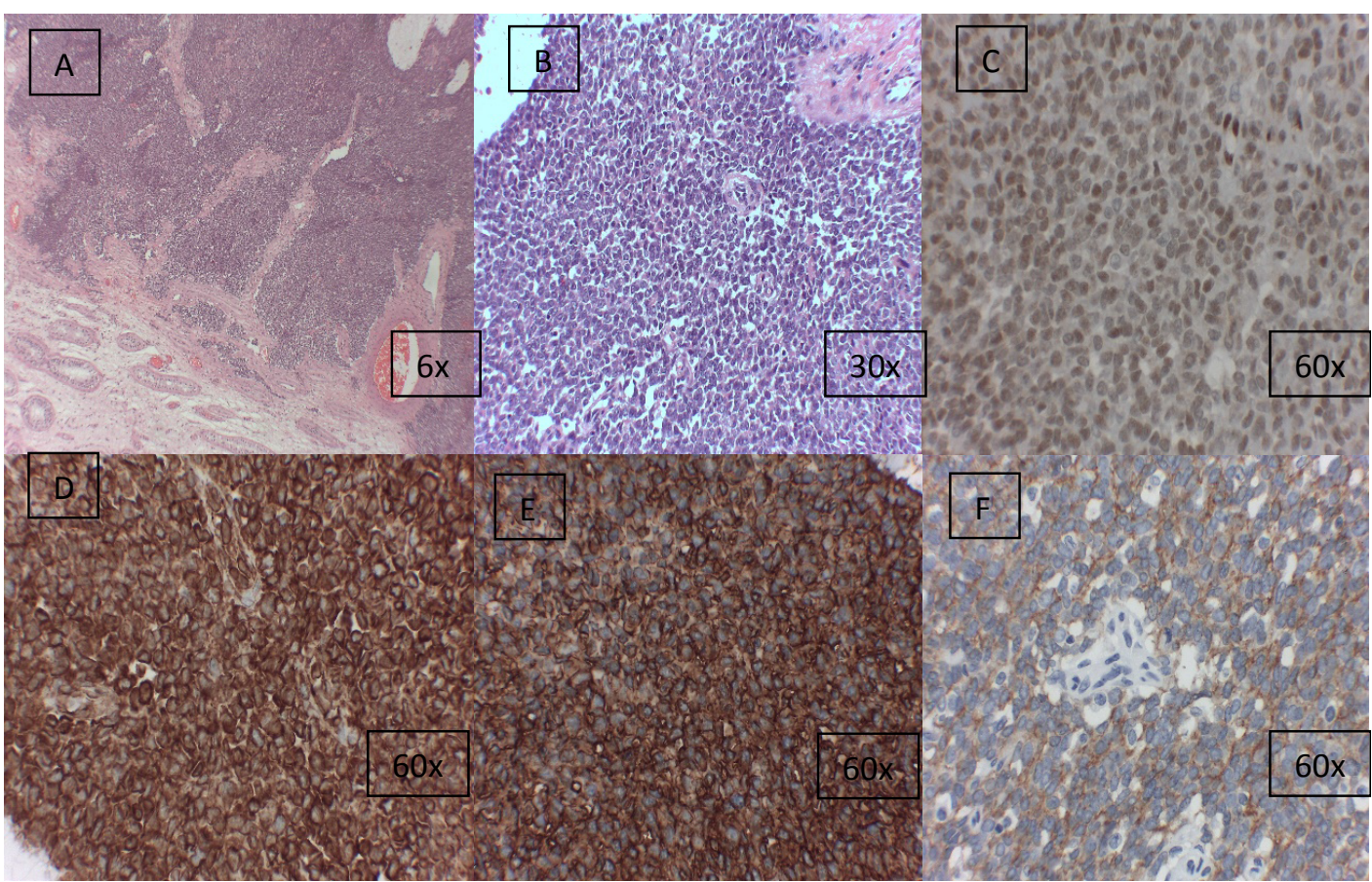

a) Haematoxylin and eosin staining showing a very celular solid tumor, divided into irregular masses by fibrous bands. At the lower end, healthy testicular parenchyma is identified. MO 6x. b) Haematoxylin and eosin staining. Neoplasm consisting of small, uniform, rounded or scarcely oval cells of poorly-defined contours. Round nucleus with frequent indentations and small nucleolus. MO 30x. c) Weak / moderate nuclear staining for Fli-1. MO 60 x. d) Intense membrane staining for CD99. MO 60x. e) Intense membrane / cytoplasmic staining for Vimentin. MO 60x. f) Moderate membrane staining for c-kit (CD117). MO 60x

Figure 2. Tumor histology samples. Morphological characteristics and immuno-staining are shown. Optical microscopic amplification at right lower corner of each picture is shown.

\section{Additional medical tests}

- Thorax X ray: No evidence of metastatic disease.

- CT thorax-abdomen-pelvis (01.23.2015): Just highlight the presence of a left para-aortic lymphadenomegaly of $1.3 \mathrm{~cm}$ of bigger diameter, non- clearly pathological. Axillary and inguinal lymphadenomegalies considered as reactive ones.

- Blood test with common tumor markers: general biochemistry, hemogram and coagulation were in normal range. Lactate dehydrogenase (LDH), $\alpha$ - fetoprotein ( $\alpha-\mathrm{FP})$ and $\beta$ - Human Chorionic Gonadotropin ( $\beta-\mathrm{HCG}$ ) were negative.

\section{Adjuvant treatment}

\section{a) Systemic therapy}

Before considering any adjuvant systemic therapy, the medical oncologist requested a review of the pathology samples of orchiectomy, modifying the pathology diagnosis to an Ewing Sarcoma of the testis, supported by the finding of EWS gene rearrangement (Figure 3).

Extension work-up (CT scan and PET-CT) was repeated which were negative for metastasis.

A poly-chemotherapy scheme based on combination of common drugs used in Ewing sarcoma (vincristine $(\mathrm{V})$, actinomycin D (A), cyclophosphamide (C), ifosfamide (I) and etoposide (E) is proposed. From March 2015 to February 2016, the patient received a total of 7 cycles of VAC and 7 cycles of IE. The patient suffered Grade IV febrile neutropenia as the mayor toxicity, according to the latest version of NCI CTCAE [8].

\section{b) Local treatment: radiotherapy}

After finishing systemic therapy, the patient was referred to the 


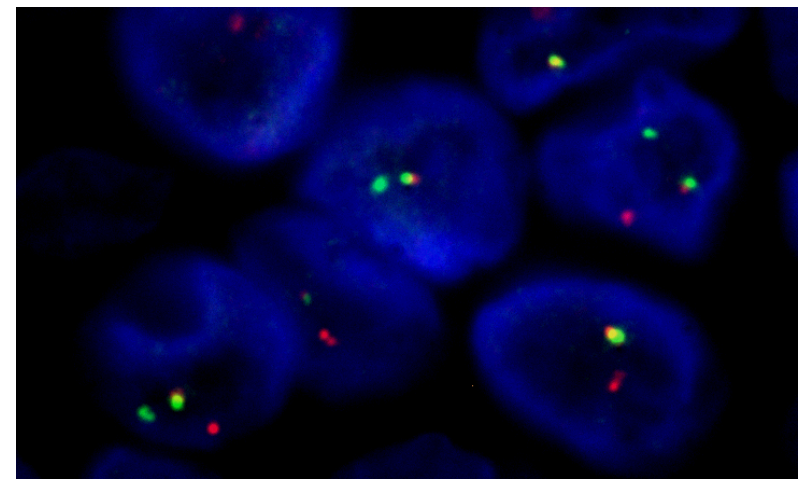

Figure 3. EWS rearrangement shown by Fluorescence In Situ Hybridization (FISH) technique. A Dual Color Break Apart specific locus probe was used to study the rearrangement of the EWSR1 gene, located in the 22q12.2 chromosomal region. The probe consists of two colors, a green signal and a red signal that mark the 5 'and 3 ' ends of the gene, respectively. In the absence of translocation, the two signals will appear together, and separated in the presence of alteration, as shown in this case.

\section{Radiation Oncology Department for treatment assessment.}

Given the limited published evidence on the role of radiotherapy specifically on this entity and location, treatment was indicated based on the management of other testicular and para-testicular tumours of mesenchymal lineage and also according to Skeletal and EES guidelines, in which surgical bed may be at risk of microscopical seeding, which may have happened in our case. A new CT scan prior to radiation confirmed the absence of macroscopic local residual disease or metastasis.

Planning CT and immobilization devices: With the patient in supine position, with arms above his head and legs in "frog" position, a mold care pillow from mid back to mid legs, was used to hold the testis and to ensure daily reproducibility. A high density bolus (eXaSkin;, Anatomical Geometry, S.L. Mairena del Aljarafe, Seville, Spain) was also used to assure a proper radiation dose distribution. A planning CT scan (Toshiba Aquillion) with $3 \mathrm{~mm}$ thick slices was done from upper abdomen to mid legs (Figure 4).

Treatment volumes: Using the Eclipse ${ }^{\mathrm{m} m}$ Treatment Planning System (Varian Medical Systems, Palo Alto, California, USA), a Clinical Target Volume (CTV) was generated to encompass the orchiectomy surgical bed, including the left scrotum, left inguinal cord and ipsilateral pubic subcutaneous tissue without local nodal stations. A planning target volume (PTV) was added by a $0.5 \mathrm{~cm}$ extension in all directions (Figure 4).

Dose prescription to target volume: A dose of $45 \mathrm{~Gy}$ in $1.8 \mathrm{~Gy} /$ fraction was prescribed to the $100 \%$ of the CTV, according to clinical guidelines [9]. To ensure a proper dose distribution with optimal CTV and PTV coverage, radiation physicists made use of Volumetric Modulated Arch Therapy (VMAT) with 2 arches. In figure 5, isodose lines distribution are shown in the planning CT scan (axial). Despite the proximity of right testis, we were able to obtain an optimal dose distribution thanks to the abovementioned technique.

\section{Outcome}

Figure 6 shows skin toxicity 2 weeks after radiotherapy ending. At the last follow- up (6 months after RT ending, and 24 months after surgical resection), there is no evidence of loco-regional or distant relapse. Overall, the three treatment modalities were well tolerated. Partial alopecia in the treatment field as midterm toxicity. No urinary, rectal or sexual problems related to any treatment used.

\section{Discussion}

Despite the abovementioned low incidence of testicular neoplasm in the general population, according to recent published data from cancer databases from different European countries, in males aged 25-49, testicular cancer is the most common cancer, accounting for around $14 \%$ of all cases [10].

Histologic classification of these tumors is complex, and includes seminomas, choriocarcinoma, and a variety of teratomas, germ cell tumors and other entities [2].

By using the MeSH tool in PubMed.gov using as key words "testicular neoplasms," "Ewing Sarcoma" and "PNET", we found only 4 clinical cases in which the diagnosis of Ewing's sarcoma at testicular level are reported. The first one, reported in an article published in 1983, described a post-mortem pathology diagnosis of a testicular PNET with wide spread throughout the body in a 30 year -old man [11]. The second one, reported in a correspondence letter, is a 21 year -old man, who debuted with back pain in the context of vertebral body L1 metastasis from a primary of the testis. It was found to have bone

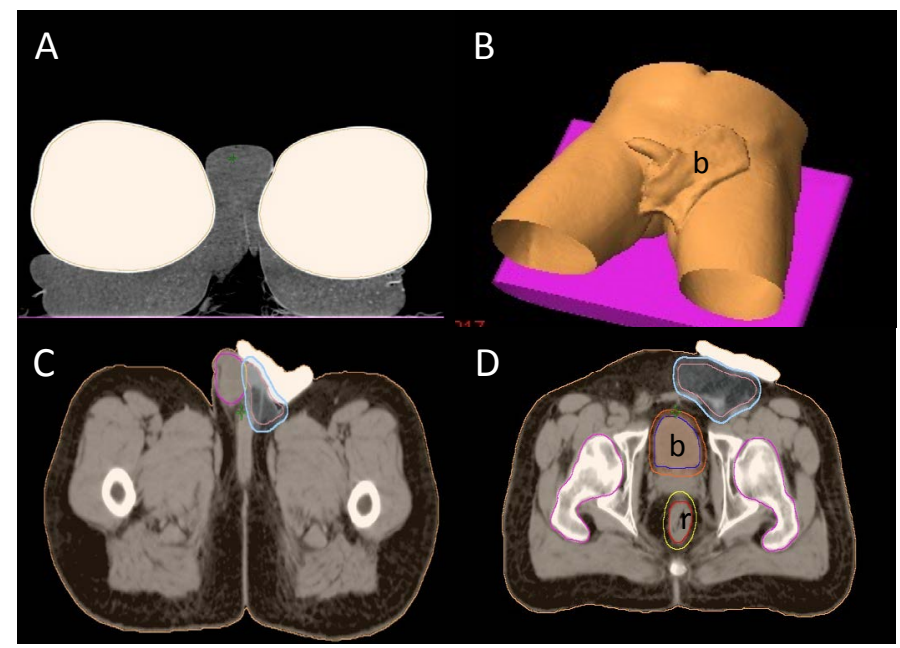

Figure 4. A) Axial slice showing the immobilization device (Mold Care $\left.{ }^{\circledR}\right)(\mathrm{m})$. B) 3D reconstruction of the bolus displacement (b). C) CT scan Axial section at testis level. Pink line corresponds to the Clinical Target volume (CTV). Blue line represents the planning target volume (PTV). D) CT scan axial section at femoral heads level. Bladder (b) and rectum $(r)$ are represented.

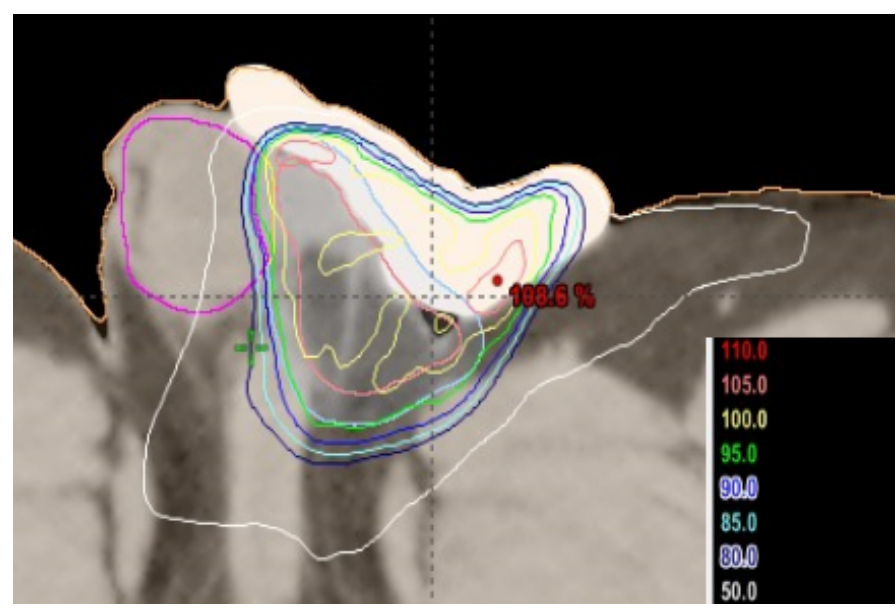

Figure 5. Axial CT scan slice at testicular level. Dosimetry planning. Table at the bottom right corner shows different isodose percentage represented by different colours. Right testis in pink (RT) 


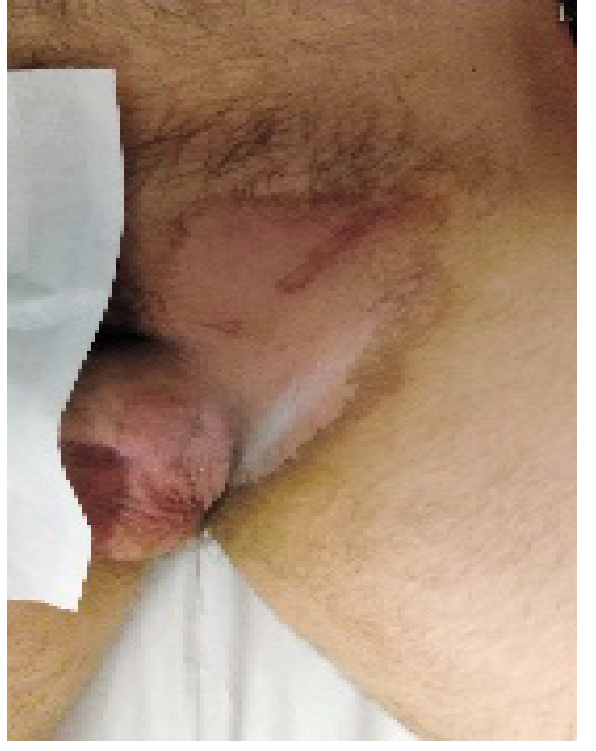

Figure 6. Skin toxicity 2 weeks after RT ending. Grade III dermatitis in resolution.

marrow involvement and was considered stage IV as initial staging. After orchiectomy, he received chemotherapy (scheme not showed). No surveillance was reported. The paper is mainly focused on pathologic review and differential diagnostic of testicular and para-testicular small cell neoplasm [12]. The third case was reported in 2013 in Germany. It showed an extensive pathological review of a testicular PNET in a 25 year-old man, with scarce information about clinical aspects [13]. The last case was published in 2015 in a Japanese scientific journal and written in Japanese. It described the case of a 44 year- old patient with a 4- year history of progressive growth of testicular volume. It was first diagnosis of a non PNET tumor in advanced stage. After a second pathologic review, they reported a primitive neuroectodermal tumor in the context of teratoma. After several chemotherapy cycles and radiotherapy, the patient was exitus [14]. In just one of the previous reported cases, EWS gene rearrangement was found.

\section{Conclusions}

This case comes to enlarge the scarce experience previously published about this weird entity. To our knowledge, this is the $5^{\text {th }}$ case of Ewing sarcoma of the testis that has been published in the literature. We believe it will be of great interest and will help other clinicians in their daily clinical decisions, regarding diagnostic, surgical, medical and radiation oncology approaches. According with recent evidencebased European and North American clinical guidelines, we want to highlight the importance of a second pathology review at medical centres with multidisciplinary expert committees, which may influence the treatment and prognosis of these unusual cases [15].

\section{Consent}

Written and oral informed consent was obtained from the patient for publication of this case report and accompanying images. A copy of the written consent is available for review by the Editor-in-Chief of this journal.

\section{References}

1. La Vecchia C, Bosetti C, Lucchini F, Bertuccio P, Negri E, et al. (2010) Cance mortality in Europe, 2000-2004, and an overview of trends since 1975. Ann Oncol 21: 1323-1360. [Crossref]

2. Albers P, Albrecht W, Algaba F, Bokemeyer C, Cohn-Cedermark G, et al. (2015) Guidelines on Testicular Cancer: 2015 Update. Eur Urol 68: 1054-1068. [Crossref]

3. Turc-Carel C, Aurias A, Mugneret F, Lizard S, Sidaner I, et al. (1988) Chromosomes in Ewing's sarcoma. I. An evaluation of 85 cases of remarkable consistency of $t(11 ; 22)$ (q24;q12). Cancer Genet Cytogenet 32: 229-238. [Crossref]

4. Zucman J, Melot T, Desmaze C, Ghysdael J, Plougastel B, et al. (1993) Combinatoria generation of variable fusion proteins in the Ewing family of tumours. EMBO J 12 : 4481-4487. [Crossref]

5. Ahmad R, Mayol BR, Davis M, Rougraff BT (1999) Extraskeletal Ewing's sarcoma. Cancer 85: 725-731. [Crossref]

6. Applebaum MA1, Worch J, Matthay KK, Goldsby R, Neuhaus J, et al. (2011) Clinical features and outcomes in patients with extraskeletal Ewing sarcoma. Cancer 117: 3027 3032. [Crossref]

7. Lee J, Hoang BH, Ziogas A, Zell JA (2010) Analysis of prognostic factors in Ewing sarcoma using a population-based cancer registry. Cancer 116: 1964-1973. [Crossref]

8. National Cancer Institute. Common Terminology Criteria for Adverse Events v4.0. NCI, NIH, DHHS. May 29, 2009. NIH publication \# 09-7473.

9. Donaldson SS (2004) Ewing sarcoma: radiation dose and target volume. Pediatr Blood Cancer 42: 471-476. [Crossref]

10. Hippisley-Cox J, Coupland C (2013) Symptoms and risk factors to identify men with suspected cancer in primary care: derivation and validation of an algorithm. Br J Gen Pract 63: e1-10. [Crossref]

11. Aguirre P, Scully RE (1983) Primitive neuroectodermal tumor of the testis. Report of a case. Arch Pathol Lab Med 107: 643-645. [Crossref]

12. Masoom S, Venkataraman G, Hammadeh R, Wojcik EM (2007) Ewing's sarcoma/ primitive neuroectodermal tumour in a possible primary testicular location. Correspondence letter. Pathology 39: 599-601. [Crossref]

13. Brandt S, Lohe B, Vogetseder A, Rüdiger T, Moch H, et al. (2013) Primitive neuroectodermal tumor of the testis. Molecular analysis and discussion of genesis. Pathologe 34: 70-73. [Crossref]

14. Goto T, Sato M, Fujii S, Itoh J, Soma F (2015) [A CASE OF PRIMITIVE NEUROECTODERMAL TUMOR OF THE TESTIS]. Nihon Hinyokika Gakka Zasshi 106: 53-58. [Crossref]

15. ESMO / European Sarcoma Network Working Group (2012) Soft tissue and viscera sarcomas: ESMO Clinical Practice Guidelines for diagnosis, treatment and follow-up. Ann Oncol 23: vii 92-99. [Crossref]

Copyright: (C2017 Gordillo MJO. This is an open-access article distributed under the terms of the Creative Commons Attribution License, which permits unrestricted use, distribution, and reproduction in any medium, provided the original author and source are credited. 